UDC 614.7

DOI: $10.21668 /$ health.risk/2021.2.06.eng

Research article

\title{
ASSESSING ACUTE INHALATION HEALTH RISK CAUSED BY EXPOSURE TO PRODUCTS CREATED BY NICOTINE-CONTAINING STUFF CONSUMPTION IN ENCLOSED SPACES
}

\author{
E.V. Zaritskaya ${ }^{1,2}$, V.N. Fedorov ${ }^{1,2}$, I.S. Iakubova ${ }^{2}$ \\ ${ }^{1}$ North-West Scientific Center for Hygiene and Public Health, 4 2-ya Sovetskaya Str., Saint Petersburg, 191036, \\ Russian Federation \\ ${ }^{2}$ North-Western State Medical University named after I.I. Mechnikov, 41 Kirochnaya Str., Saint Petersburg, \\ 195067, Russian Federation
}

Contemporary research reveals that electronic devices for nicotine consumption produce not so negative effects on health due to adverse chemicals being emitted in substantially lower quantities. Nevertheless, such consumption still results in emission of various organic and non-organic substances with their effects on health being rather unpredictable. It is necessary to conduct additional studies, including those focusing on passive smoking of electronic cigarettes and assessing health risks caused by exposure to them.

Our research goal was to assess acute health risks caused by passive consumption of tobacco and nicotinecontaining products.

We built a model for tobacco or nicotine consumption by actual consumers (volunteers) and the process was evened as per a number of consumption sessions. We examined three products: tobacco cigarettes (cigarettes), electronic nicotine delivery system (ENDS), and a tobacco heating system (IQOS). Background air quality parameters were measured in a specifically organized enclosed space prior to each study session. We also conducted experiments in so called «reference groups» when research participants didn't consume the examined products but were in the same conditions. Health risks were assessed as per the Guide $R$ 2.1.10.1920-04 «The Guide on assessing health risks caused by exposure to chemicals that pollute the environment».

Use of ENDS and IQOS does not result in significant changes in air composure and does not cause unacceptable acute health risk. Combined effects produced by contaminants on organs and systems resulted in health risks for respiratory organs, eyes, and body as a whole being insignificantly higher than permissible levels; these risks were practically the same as those detected for a reference group. Tobacco smoking resulted in unacceptable acute risks 1.5 hours after an experiment started; these risks were caused by elevated concentrations of such contaminants as acetaldehyde, formaldehyde, $P M_{2.5}, P M_{10}$, and carbon monoxide.

Key words: health risk, acute risk, acute additional risk, passive smoking, tobacco, nicotine-containing products, cigarettes, electronic nicotine delivery systems, electronic tobacco heating devices.

According to data provided by the RF Public Healthcare Ministry, in Russia annually up to 300 thousand people die due to smokingrelated diseases $[1,2]$. As per data provided by the WHO, tobacco smoking is among the most significant threats for human health that causes approximately 7 million death cases every year all over the world; 890 thousand out of them are non-smokers who were exposed to tobacco smoke $[3,4]$.

According to the Concept on the state policy aimed at fighting against consumption of tobacco and other nicotine-containing products for a period up to $2035^{1}$, the RF Public Health-

(C) Zaritskaya E.V., Fedorov V.N., Iakubova I.S., 2021

Ekaterina V. Zaritskaya - Head of the Laboratory Research Department, post-graduate student at the Department for Preventive Medicine and Health Protection (e-mail: zev-79@mail.ru; tel.: +7 (812) 717-96-43; ORCID: http://orcid.org/0000-0003-2481-1724).

Vladimir N. Fedorov - Researcher at the Department for Analysis, Assessment, and Prediction of the Environment and Population Health in the Arctic regions in the Russian Federation; Junior researcher (e-mail: vf1986@mail.ru; tel.: +7 (812) 717-01-54; ORCID: http://orcid.org/0000-0003-1378-1232).

Irek S. Iakubova - Doctor of Medical Sciences, Professor at the Department for Preventive Medicine and Health Protection (e-mail: yakubova-work@yandex.ru; tel.: +7 (812) 543-17-47; ORCID: https://orcid.org/0000-0003-2437-1255).

${ }^{1}$ The Concept on the state policy aimed at fighting against consumption of tobacco and other nicotine-containing products for a period up to 2035 and further: The RF Government Order dated November 18, 2019 No. 2732-r. Garant: the information and legal portal. Available at: https://www.garant.ru/products/ipo/prime/doc/72943536/(January 27, 2021). 
care Ministry aims to reach maximum possible decrease in mortality and morbidity caused by smoking-related diseases as well as to prevent nicotine-containing products from further spread among population. To achieve the goals set by the Concept a system for managing its implementation is being created; this system involves developing mechanisms for risk monitoring, assessment, and minimization.

According to the Concept, by 2035 a number of smokers among adult population should go down to $21 \%$. Initial parameters are fixed as in December 2018 when smokers accounted for $29 \%$ among adult population in Russia.

It should be noted that recently new smoking products have been becoming more and more popular among population, namely electronic nicotine delivery systems (ENDS) and electronic tobacco heating systems (ETHS) $[5,6]$. It is especially true for young people aged 18-24 since approximately $19.1 \%$ people in this age group use such products and it is 10 times higher than in other age groups [7]. Electronic vaping is already widely spread all over the world and many countries introduce strict rules for it given new available actual data on negative effects produced by vaping on health [8-12]. But at present it is still rather difficult to issue legislative acts regarding a ban on use of electronic cigarettes in public places; although such an initiative was introduced to the State Duma, an absence of evidence base proving that "passive consumption" of nicotine-containing products causes health risks doesn't allow determining what limitations should be imposed on distribution of such products which are considered an alternative to tobacco products [13-21].

Besides, there were some studies [22] on electronic cigarettes and a medicinal nicotine inhalator as an etalon product performed in the USA, Great Britain and Poland; they revealed that ENDS produced certain toxicants but their levels were from 9 to 450 times lower than in cigarette smoke. But still, a mix that was inhaled via using ENDS contained small quantities of 1,2-propanediol, 1,2,3-propanetriol, diacetyl, flavor additives, and also nicotine in trace quantities [23].

Given all the above mentioned, additional studies are required including those focusing on "passive consumption" of electronic cigarettes; it is also vital to assess health risks caused by exposure to them.

Our research goal was to assess acute health risk caused by passive consumption of tobacco in nicotine-containing products.

Data and methods. 3 types of nicotinecontaining products were examined as sources of adverse chemicals emission, namely cigarettes, an electronic nicotine delivery system (ENDS), and an electronic tobacco heating system (ETHS). Volunteers that had been smoking for not less than three years prior to our experiment took part in it; they all gave their informed consent to participate. To achieve greater representativeness in our research, we examined each product for three days and each examination involved three volunteers participating in it. A reference group was made up of non-smokers who stayed in an experiment room for four and a half hours. All examinations were performed in a specifically prepared room.

Background air quality was estimated prior to the experiment started. Air samples were taken thrice during the experiment, 1.5, 3 and 4.5 hours after it had started. Sampling devices and a meteometer gauge were placed at a height where a sitting person breathed.

Air samples were analyzed at "Arbitrazh" certified laboratory chemical and analytical center in conformity with the requirements fixed in the valid regulatory and methodical documents ${ }^{2}$. All obtained results were statistically processed with IBM SPSS Statistics, v. 22.

\footnotetext{
${ }^{2}$ Methodical guidelines 4.1.1673-03. Chromato-mass-spectrometric determination of substances that are contained in tobacco and tobacco dust in ambient air; M-21. The procedure for measuring nicotine mass concentration in industrial emissions into ambient air, in working area air and in ambient air, with gas chromatography; M-MVI-198-07. The procedure for measuring carbonyl-containing substances in ambient air in settlements and working area air via HPLC using active sampling device; $\mathrm{RD}$ 52.04.830-2015. Mass concentration of particulate matter $\mathrm{PM}_{10}$ and $\mathrm{PM}_{2,5}$ in ambient air. The gravimetric procedure; "Methodical guidelines on analyzing objects with unknown structure with GLC, CMS, HPLC, GC/IR/PS, AE-ICP, MS-ICP" D.I. Mendeleev's Russian Metrology Scientific and Research Institute No. 01-07. KonsultantPlus. Available at: https:// docs.cntd.ru (January 26, 2021).
} 
Our research was given the following methodical grounds: the Guide R 2.1.10.1920-04 "The Guide on assessing health risks under exposure to chemicals that pollute the environment"3, guidelines developed by the US Environmental Protection Agency ${ }^{4}$ and the Office of Environmental Health Hazard Assessment ${ }^{5}$, Integrated Risk Information System, (IRIS).

Risks were assessed by an organization certified in this sphere in the System for voluntary certification of institutions dealing with health risk assessment (Conformance Certificate No. SDS 062, registered in the System database on December 26, 2018).

We considered short-term (acute) exposure lasting for several hours in our research. This scenario was selected due to the following factors:

- the experiment involved modeling an exposure scenario taking into account potential inhalation of contaminant with air in a closed room (so called "passive consumption") and not its direct (or "active") consumption;

- "passive consumers" had to spend limited amount of time in the experiment room according to the experiment design;

- there was no possibility to predict periodicity of contacts an exposed person would have with the examined substances in real conditions during the whole life span; consequently, it was impossible to calculate chronic dose exposure $[11,16,17,19]$.

As for this experimental situation, we modeled 4.5-hour exposure to contaminants in the air in the closed experimental room and it was considered short-term and acute exposure.

Reference concentrations under acute inhalation exposure, ARfC, were applied to assess acute risks caused by exposure to contaminants. Their values were taken from the Appendix 2 to the Guide R 2.1.10.1920-04 as well as from USEPA and OEHHA databases.
We applied a standard formula for calculating an acute risk (hazard quotient $\mathrm{HQ}_{\mathrm{R}}$ ) in our research:

$$
H Q_{R}=C / A R f C,
$$

where $C$ is concentration of a substance, $A R f C$ is a reference concentration under acute exposure.

In addition to acute risk assessment and according to the Guide R 2.1.10.1920-04 we also calculated some parameters that described difference between an acute risk caused by exposure to a certain chemical among people who consumed tobacco or nicotine at a specific moment of time during the experiment and an acute risk that occurred for the reference group at the same moment. A term "acute additional risk" $\left(H Q_{R \_a d d}\right)$ was applied for this parameter in the present work; it was calculated as per the following formula:

$$
H Q_{R \_a d d}=H Q_{R \_t}-H Q_{R \_b k g d},
$$

where $H Q_{R_{-} t}$ is an acute risk at a certain moment of time during the experiment (when samples were being taken); $H Q_{R_{-} b k g d}$ is a background acute risk for the reference group at the same moment of time during the experiment.

Results and discussion. The hazard identification stage involved estimating a structure of contaminants in the air in the closed room that occurred due to different tobacco- and nicotine-containing products consumption (Table 1).

Acute health risks were assessed basing on results obtained via laboratory tests performed on the air in the closed room; the assessment revealed that air quality became unacceptable already after 1.5 hours when cigarette smoking was modeled in the experiment. It was primarily due to elevated concentrations of acetaldehyde, formaldehyde, and particulate matter that

${ }^{3} \mathrm{R}$ 2.1.10.1920-04. The Guide on assessing health risks caused by exposure to chemicals that pollute the environment. Moscow, The Federal Center for State sanitary and Epidemiologic Surveillance of the RF Public Healthcare Ministry Publ., 2004, $143 \mathrm{p}$.

${ }^{4}$ Location-Specific Environmental Information. United States Environmental Protection Agency (US EPA). Available at: https://www.epa.gov/environmental-topics/location-specific-environmental-information (January 26, 2021).

${ }^{5}$ Environmental Topics. The Office of Environmental Health Hazard Assessment (OEHHA). Available at: https://oehha.ca.gov/environmental-topics (January 26, 2021). 
were 1.5-2 times higher than permissible ones after 1.5 hours. Acute risks caused by acetaldehyde and formaldehyde contents didn't exceed permissible levels during the whole experiment when ENDS and ETHS consumption was modeled (Tables 2 and 3).

Overall, we can state that air contamination in the closed room caused by ENDS and ETHS consumption didn't cause unacceptable acute health risks even after a long period of time spent in that room without any air ventilation; meanwhile, cigarette smoking caused unacceptable health risk already 1.5 hour after the experiment started (Table 2).

Taking into account data on effects produced by various chemicals on a body as a whole or organs and systems in particular provided in Appendix 2, the Guide R 2.1.10.192004 , we created a list of critical organs and systems that were target ones for adverse impacts exerted by the examined chemicals (Table 4).
Table 1

Priority contaminants in the air in the experimental room and hygienic standards regulating them

\begin{tabular}{|l|c|}
\hline \multicolumn{1}{|c|}{ Contaminant } & ARfC, $\mathrm{mg} / \mathrm{m}^{3}$ \\
\hline Formaldehyde & 0.048 \\
\hline Acetaldehyde & 0.115 \\
\hline Buta-1,3-dien (Divinyl) & 0.11 \\
\hline Benzene & 0.15 \\
\hline Methylbenzene (Toluene) & 3.8 \\
\hline Carbon oxide & 23 \\
\hline Nitrogen dioxide (Nitrogen (IV) oxide) & 0.47 \\
\hline Nitrogen (II) oxide (Nitrogen oxide) & 0.72 \\
\hline PM $_{2,5}$ & 0.065 \\
\hline PM $_{10}$ & 0.15 \\
\hline
\end{tabular}

Note :

$\mathrm{ARfC}$ are reference concentrations under acute exposure;

$\mathrm{PM}_{10}$ are particulate matter with mass concentrations of particles smaller than $10 \mu \mathrm{m}$ in diameter;

$\mathrm{PM}_{2,5}$ are particulate matter with mass concentrations of particles smaller than $2.5 \mu \mathrm{m}$ in diameter.

Table 2

Concentrations of contaminants and acute and acute additional risks caused by specific contaminants occurring due to tobacco or nicotine consumption in the air in the closed room at different moments of time during the experiment

\begin{tabular}{|c|c|c|c|c|c|c|c|c|c|c|c|c|}
\hline \multirow{3}{*}{ Components } & \multirow{3}{*}{ Experiment } & \multicolumn{11}{|c|}{ Concentrations (average values, $\mathrm{mg} / \mathrm{m}^{3}$ ) and risk levels } \\
\hline & & \multicolumn{2}{|c|}{$\begin{array}{l}\text { Background } \\
\text { values (sam- } \\
\text { pling time } \\
8: 00-9: 30 \text { ) }\end{array}$} & \multicolumn{3}{|c|}{$\begin{array}{c}\text { After } 1.5 \text { hours } \\
\text { (sampling time } \\
9: 40-11: 10 \text { ) }\end{array}$} & \multicolumn{3}{|c|}{$\begin{array}{l}\text { After } 3 \text { hours } \\
\text { (sampling time } \\
11: 20-12: 50 \text { ) }\end{array}$} & \multicolumn{3}{|c|}{$\begin{array}{c}\text { After } 4.5 \text { hours } \\
\text { (sampling time } \\
13: 00-14: 30 \text { ) }\end{array}$} \\
\hline & & $\begin{array}{l}\text { Concen- } \\
\text { tration }\end{array}$ & $\begin{array}{l}\text { Acute } \\
\text { risk }\end{array}$ & $\begin{array}{c}\text { Concen- } \\
\text { tration }\end{array}$ & $\begin{array}{l}\text { Acute } \\
\text { risk }\end{array}$ & \begin{tabular}{|c|} 
Acute \\
additional \\
risk \\
\end{tabular} & $\begin{array}{c}\text { Concen- } \\
\text { tration }\end{array}$ & $\begin{array}{l}\text { Acute } \\
\text { risk }\end{array}$ & \begin{tabular}{|c|} 
Acute \\
additional \\
risk \\
\end{tabular} & $\begin{array}{c}\text { Concen- } \\
\text { tration }\end{array}$ & $\begin{array}{c}\text { Acute } \\
\text { risk }\end{array}$ & \begin{tabular}{|c|} 
Acute \\
additional \\
risk \\
\end{tabular} \\
\hline 1 & 2 & 3 & 4 & 5 & 6 & 7 & 8 & 9 & 10 & 11 & 12 & 13 \\
\hline \multirow{4}{*}{ Benzene } & Reference & 0.0011 & 0.007 & 0.0016 & 0.01 & & 0.011 & 0.07 & & 0.009 & 0.06 & \\
\hline & Cigarettes & 0.0009 & 0.006 & 0.014 & 0.09 & 0.083 & 0.027 & 0.18 & 0.107 & 0.034 & 0.23 & 0.17 \\
\hline & ENDS & 0.0009 & 0.006 & 0.0022 & 0.02 & 0.004 & 0.0029 & 0.02 & -0.054 & 0.0029 & 0.02 & -0.04 \\
\hline & ETHS & 0.0013 & 0.009 & 0.0015 & 0.01 & -0.001 & 0.0015 & 0.01 & -0.063 & 0.0018 & 0.01 & -0.05 \\
\hline \multirow{4}{*}{ Toluene } & Reference & 0.0034 & 0.001 & 0.0053 & 0.001 & & 0.0054 & 0.001 & & 0.0052 & 0.001 & \\
\hline & Cigarettes & 0.0019 & 0.001 & 0.023 & 0.01 & 0.005 & 0.041 & 0.01 & 0.009 & 0.053 & 0.01 & 0.01 \\
\hline & ENDS & 0.0022 & 0.001 & 0.0057 & 0.002 & 0 & 0.0083 & 0.002 & 0.001 & 0.0088 & 0.002 & 0.001 \\
\hline & ETHS & 0.0044 & 0.001 & 0.0045 & 0.001 & 0 & 0.004 & 0.001 & 0 & 0.0051 & 0.001 & 0 \\
\hline \multirow{4}{*}{ Formaldehyde } & Reference & 0.024 & 0.5 & 0.035 & 0.73 & & 0.038 & 0.79 & & 0.044 & 0.92 & \\
\hline & Cigarettes & 0.026 & 0.542 & 0.072 & 1.50 & 0.771 & 0.1 & 2.08 & 1.292 & 0.106 & 2.21 & 1.29 \\
\hline & ENDS & 0.024 & 0.5 & 0.033 & 0.69 & -0.042 & 0.042 & 0.88 & 0.083 & 0.044 & 0.92 & 0.00 \\
\hline & ETHS & 0.022 & 0.458 & 0.029 & 0.60 & -0.125 & 0.031 & 0.65 & -0.146 & 0.036 & 0.75 & -0.17 \\
\hline \multirow{4}{*}{ Acetaldehyde } & Reference & 0.008 & 0.07 & 0.014 & 0.12 & & 0.017 & 0.15 & & 0.018 & 0.16 & \\
\hline & Cigarettes & 0.009 & 0.078 & 0.125 & 1.09 & 0.965 & 0.262 & 2.28 & 2.13 & 0.332 & 2.89 & 2.73 \\
\hline & ENDS & 0.008 & 0.07 & 0.017 & 0.15 & 0.026 & 0.03 & 0.26 & 0.113 & 0.248 & 2.16 & 2.00 \\
\hline & ETHS & 0.009 & 0.078 & 0.02 & 0.17 & 0.052 & 0.031 & 0.27 & 0.122 & 0.042 & 0.37 & 0.21 \\
\hline \multirow{4}{*}{$\begin{array}{l}\text { Particulate } \\
\text { matter } \mathrm{PM}_{2,5}\end{array}$} & Reference & 0.034 & 0.523 & 0.048 & 0.74 & & 0.044 & 0.68 & & 0.053 & 0.82 & \\
\hline & Cigarettes & 0.026 & 0.4 & 0.68 & 10.46 & 9.723 & 1.2 & 18.46 & 17.785 & 1.2 & 18.46 & 17.65 \\
\hline & ENDS & 0.045 & 0.692 & 0.065 & 1.00 & 0.262 & 0.078 & 1.20 & 0.523 & 0.084 & 1.29 & 0.48 \\
\hline & ETHS & 0.039 & 0.6 & 0.071 & 1.09 & 0.354 & 0.071 & 1.09 & 0.415 & 0.07 & 1.08 & 0.26 \\
\hline
\end{tabular}




\begin{tabular}{|c|c|c|c|c|c|c|c|c|c|c|c|c|}
\hline 1 & 2 & 3 & 4 & 5 & 6 & 7 & 8 & 9 & 10 & 11 & 12 & 13 \\
\hline \multirow{4}{*}{$\begin{array}{c}\text { Particulate } \\
\text { matter } \\
\mathrm{PM}_{10}\end{array}$} & Reference & 0.048 & 0.32 & 0.042 & 0.28 & & 0.047 & 0.31 & & 0.0513 & 0.34 & \\
\hline & Cigarettes & 0.035 & 0.233 & 0.66 & 4.40 & 4.12 & 1.2 & 8.00 & 7.687 & 1.1333 & 7.56 & 7.21 \\
\hline & ENDS & 0.053 & 0.353 & 0.066 & 0.44 & 0.16 & 0.097 & 0.65 & 0.333 & 0.0903 & 0.60 & 0.26 \\
\hline & ETHS & 0.038 & 0.253 & 0.073 & 0.49 & 0.207 & 0.075 & 0.50 & 0.187 & 0.0747 & 0.50 & 0.16 \\
\hline \multirow{4}{*}{$\begin{array}{l}\text { Carbon } \\
\text { oxide }\end{array}$} & Reference & 0.467 & 0.648 & 0.967 & 1.34 & & 0.967 & 1.34 & & 1.167 & 1.62 & \\
\hline & Cigarettes & 0.367 & 0.509 & 8.167 & 11.34 & 10 & 10.633 & 14.77 & 13.426 & 13.333 & 18.52 & 16.90 \\
\hline & ENDS & 0.467 & 0.648 & 1.9 & 2.64 & 1.296 & 2.967 & 4.12 & 2.778 & 3.3 & 4.58 & 2.96 \\
\hline & ETHS & 0.467 & 0.648 & 1.167 & 1.62 & 0.278 & 1.033 & 1.44 & 0.093 & 1.2 & 1.67 & 0.05 \\
\hline \multirow{4}{*}{$\begin{array}{c}\text { Nitrogen } \\
\text { (II) oxide } \\
\text { (Nitrogen } \\
\text { oxide) }\end{array}$} & Reference & 0.041 & 0.056 & 0.043 & 0.06 & 0 & 0.048 & 0.07 & 0 & 0.049 & 0.07 & 0.00 \\
\hline & Cigarettes & 0.049 & 0.068 & 0.196 & 0.27 & 0.213 & 0.293 & 0.41 & 0.34 & 0.314 & 0.44 & 0.37 \\
\hline & ENDS & 0.014 & 0.019 & 0.02 & 0.03 & -0.032 & 0.029 & 0.04 & -0.025 & 0.034 & 0.05 & -0.02 \\
\hline & ETHS & 0.053 & 0.074 & 0.053 & 0.07 & 0.013 & 0.056 & 0.08 & 0.012 & 0.056 & 0.08 & 0.01 \\
\hline \multirow{4}{*}{$\begin{array}{c}\text { Nitrogen } \\
\text { dioxide } \\
\text { (Nitrogen } \\
\text { (IV) oxi- } \\
\text { de) }\end{array}$} & Reference & 0.005 & 0.01 & 0.001 & 0.001 & 0 & 0 & 0.00 & 0 & 0 & 0.000 & 0.00 \\
\hline & Cigarettes & 0.003 & 0.006 & 0.003 & 0.01 & 0.004 & 0.002 & 0.004 & 0.004 & 0.001 & 0.003 & 0.003 \\
\hline & ENDS & 0.006 & 0.013 & 0 & 0.001 & -0.001 & 0 & 0.00 & 0 & 0 & 0.000 & 0.000 \\
\hline & ETHS & 0.012 & 0.026 & 0.001 & 0.001 & 0 & 0.002 & 0.004 & 0.004 & 0.001 & 0.001 & 0.001 \\
\hline
\end{tabular}

Table 3

Contaminants concentrations and acute risk caused by specific substances occurring due to tobacco or nicotine consumption in the air in the closed room after 4.5 hours of the experiment

\begin{tabular}{|c|c|c|c|c|c|}
\hline \multirow{2}{*}{ Components } & \multirow{2}{*}{ Experiment } & \multicolumn{2}{|c|}{ Contaminants concentrations, $\mathrm{mg} / \mathrm{m}^{3}$} & \multicolumn{2}{|c|}{ Acute risk } \\
\hline & & $M e$ & $Q_{0.25-0.75^{*}}$ & $M e^{8}$ & $Q_{0.25-0.75}{ }^{9}$ \\
\hline \multirow{4}{*}{ Formaldehyde } & Reference & 0.0365 & $0.03225-0.0395$ & 0.76 & $0.67-0.82$ \\
\hline & Cigarettes & 0.086 & $0.0605-0.1015$ & 1.79 & $1.26-2.11$ \\
\hline & ENDS & 0.0375 & $0.03075-0.0425$ & 0.78 & $0.64-0.89$ \\
\hline & ETHS & 0.03 & $0.02725-0.03225$ & 0.63 & $0.57-0.67$ \\
\hline \multirow{4}{*}{ Acetaldehyde } & Reference & 0.0155 & $0.0125-0.01725$ & 0.14 & $0.11-0.15$ \\
\hline & Cigarettes & 0.1935 & $0.096-0.2795$ & 1.68 & $0.83-2.43$ \\
\hline & ENDS & 0.0235 & $0.01475-0.0845$ & 0.20 & $0.13-0.74$ \\
\hline & ETHS & 0.0255 & $0.01725-0.03375$ & 0.22 & $0.15-0.29$ \\
\hline \multirow{4}{*}{$\begin{array}{l}\text { Buta-1,3-dien } \\
\text { (Divinyl) }\end{array}$} & Reference & 0.0002 & $0.0002-0.0002$ & 0.00 & $0.002-0.002$ \\
\hline & Cigarettes & 0.049 & $0.02705-0.0675$ & 0.45 & $0.25-0.61$ \\
\hline & ENDS & 0.001 & $0.000575-0.001525$ & 0.01 & $0.005-0.01$ \\
\hline & ETHS & 0.0004 & $0.00035-0.0004$ & 0.00 & $0.003-0.004$ \\
\hline \multirow{4}{*}{ Benzene } & Reference & 0.0053 & $0.001475-0.0095$ & 0.04 & $0.01-0.06$ \\
\hline & Cigarettes & 0.0205 & $0.010725-0.02875$ & 0.14 & $0.07-0.19$ \\
\hline & ENDS & 0.00255 & $0.001875-0.0029$ & 0.02 & $0.01-0.02$ \\
\hline & ETHS & 0.0015 & $0.00145-0.001575$ & 0.01 & $0.01-0.01$ \\
\hline \multirow{4}{*}{$\begin{array}{l}\text { Methylbenzene } \\
\text { (Toluene) }\end{array}$} & Reference & 0.00525 & $0.00475-0.005325$ & 0.001 & $0.001-0.001$ \\
\hline & Cigarettes & 0.032 & $0.017725-0.044$ & 0.01 & $0.005-0.01$ \\
\hline & ENDS & 0.007 & $0.004825-0.008425$ & 0.002 & $0.002-0.002$ \\
\hline & ETHS & 0.00445 & $0.0043-0.00465$ & 0.001 & $0.001-0.001$ \\
\hline \multirow{4}{*}{ Carbon oxide } & Reference & 0.967 & $0.842-1.017$ & 1.34 & $1.17-1.41$ \\
\hline & Cigarettes & 9.4 & $6.217-11.308$ & 13.06 & $8.63-15.71$ \\
\hline & ENDS & 2.4335 & $1.54175-3.05025$ & 3.38 & $2.14-4.24$ \\
\hline & ETHS & 1.1 & $0.8915-1.17525$ & 1.53 & $1.23-1.63$ \\
\hline \multirow{4}{*}{$\begin{array}{c}\text { Nitrogen dioxide } \\
\text { (Nitrogen (IV) oxide) }\end{array}$} & Reference & 0.0005 & $0-0.002$ & 0.0005 & $0-0.003$ \\
\hline & Cigarettes & 0.0025 & $0.00175-0.003$ & 0.005 & $0.003-0.006$ \\
\hline & ENDS & 0 & $0-0.0015$ & 0.0005 & $0-0.004$ \\
\hline & ETHS & 0.0015 & $0.001-0.0045$ & 0.003 & $0.001-0.01$ \\
\hline \multirow{4}{*}{$\begin{array}{l}\text { Nitrogen (II) oxide } \\
\text { (Nitrogen oxide) }\end{array}$} & Reference & 0.0455 & $0.0425-0.04825$ & 0.06 & $0.06-0.1$ \\
\hline & Cigarettes & 0.2445 & $0.15925-0.29825$ & 0.34 & $0.22-0.41$ \\
\hline & ENDS & 0.0245 & $0.0185-0.03025$ & 0.03 & $0.03-0.04$ \\
\hline & ETHS & 0.0545 & $0.053-0.056$ & 0.08 & $0.07-0.08$ \\
\hline
\end{tabular}


Assessing acute inhalation health risk caused by exposure to products created ...

\begin{tabular}{|c|c|c|c|c|c|}
\hline \multirow{2}{*}{ Components } & \multirow{2}{*}{ Experiment } & \multicolumn{2}{|c|}{ Contaminants concentrations, $\mathrm{mg} / \mathrm{m}^{3}$} & \multicolumn{2}{|c|}{ Acute risk } \\
\cline { 2 - 6 } & & $M e$ & $Q_{0.25-0.75}{ }^{*}$ & $M e^{8}$ & $Q_{0.25-0.75}{ }^{9}$ \\
\hline \multirow{3}{*}{$\mathrm{PM}_{2,5}$} & Reference & 0.046 & $0.0415-0.04925$ & 0.71 & $0.64-0.76$ \\
\cline { 2 - 6 } & Cigarettes & 0.94 & $0.5165-1.2$ & 14.46 & $7.95-18.46$ \\
\cline { 2 - 6 } & ENDS & 0.0715 & $0.06-0.0795$ & 1.10 & $0.92-1.22$ \\
\cline { 2 - 6 } & ETHS & 0.0705 & $0.06225-0.071$ & 1.08 & $0.96-1.09$ \\
\hline \multirow{3}{*}{$\mathrm{PM}_{10}$} & Reference & 0.0475 & $0.04575-0.048825$ & 0.32 & $0.30-0.33$ \\
\cline { 2 - 6 } & Cigarettes & 0.89665 & $0.50375-1.149975$ & 5.98 & $3.36-7.67$ \\
\cline { 2 - 6 } & ENDS & 0.07815 & $0.06275-0.091975$ & 0.52 & $0.42-0.61$ \\
\cline { 2 - 6 } & ETHS & 0.07385 & $0.06425-0.074775$ & 0.49 & $0.43-0.50$ \\
\hline
\end{tabular}

$\mathrm{N}$ o t e : Me means median; * is interquartile range.

Table 4

A list of critical organs and systems and chemicals that exert their impacts on them

\begin{tabular}{|c|c|c|}
\hline $\begin{array}{c}\text { Critical organs } \\
\text { and systems }\end{array}$ & $\begin{array}{c}\text { A number of chemicals targeting } \\
\text { a certain organ or a system }\end{array}$ & Chemicals \\
\hline Respiratory organs & 6 & $\begin{array}{c}\text { Toluene, Formaldehyde, Particulate matter } \\
\mathrm{PM}_{2,5}, \mathrm{PM}_{10}, \text { Nitrogen oxide, Nitrogen dioxide }\end{array}$ \\
\hline Nervous system & 1 & Toluene \\
\hline Development & 2 & 1,3 -butadien, Carbon monoxide \\
\hline Eyes & 3 & Toluene, Formaldehyde, Acetaldehyde \\
\hline Blood & 1 & Carbon oxide \\
\hline Impacts on a body as a whole & 2 & Particulate matter $\mathrm{PM}_{2,5}, \mathrm{PM}_{10}$ \\
\hline
\end{tabular}

Having analyzed impacts exerted by different chemicals that targeted the same organs and systems and having calculated $H I$ for them, we revealed that the highest acute risks were caused by cigarette smoking for the respiratory organs and overall impacts on a body as a whole, risk values being 28.68 and 26.02 taking background concentrations into account and 26.54 and 24.86 without them (acute additional risk) accordingly (Tables 5 and 6). We should note that acute risks for all organs and systems that were sensitive to the examined chemicals were the lowest in case ETHS was used and they practically didn't differ from those detected for the reference group.

Analysis of acute risks and acute additional risks indicated that background contamination didn't make any significant contribution into acute risks as it was confirmed by negligible differences in risk levels (Tables 5 and 6).

Despite there were several measures taken for providing purity of the experiment, we should mention several objective factors that create certain difficulties in assessing the experimental results:

1. It would be advisable to unify quantity of different consumed nicotine-containing products for proper comparative assessment of chemicals emission. However, we modeled actual tobacco or nicotine consumption by actual people who consume such products and this consumption was leveled off as per a number of consumption cases. Due to it this uncertainty can be considered negligible.

2. We detected "marker-substances" in the air in the experimental room during the experiment for the reference group and at a stage when background (initial) concentrations were determined.

3. Some "marker-substances" occur in ambient air and can influence the experimental results.

4. The air in the experimental room was not ventilated during the whole experiment so that "marker-substances" could be efficiently determined via laboratory tests. Due to it the obtained absolute values can be different from similar data obtained by other researchers.

Additional acute risks were analyzed in dynamics and this analysis revealed that the greatest additional acute risks occurred due to cigarettes smoking (Figures 1-8). Overall, consumption of all tobacco or nicotinecontaining produces resulted in additional risks during the experiment due to an increase 
Table 5

Hazard indexes for acute non-carcinogenic risk $(H I)$ caused by combined exposure to different chemicals for various organs and systems taking background concentrations into account

\begin{tabular}{|c|c|c|c|c|c|c|}
\hline \multirow{2}{*}{ Experiment } & \multicolumn{6}{|c|}{$\begin{array}{l}\text { Acute non-carcinogenic risks for specific organs and systems } \\
\text { (hazard index } H I \text { ) under 4.5-hour exposure }\end{array}$} \\
\hline & $\begin{array}{l}\text { Respiratory } \\
\text { organs }\end{array}$ & $\begin{array}{r}\text { Systemic } \\
\text { impacts }\end{array}$ & $\begin{array}{c}\text { Nervous } \\
\text { system }\end{array}$ & $\begin{array}{l}\text { Develop- } \\
\text { ment }\end{array}$ & Eyes & Blood \\
\hline Reference group (non-smokers) & 2.14 & 1.16 & 0.001 & 1.62 & 1.08 & 1.62 \\
\hline Cigarette smoking & 28.68 & 26.02 & 0.014 & 18.52 & 5.11 & 18.52 \\
\hline ENDS consumption & 2.86 & 1.89 & 0.002 & 4.58 & 3.08 & 4.58 \\
\hline ETHS consumption & 2.41 & 1.58 & 0.001 & 1.67 & 1.12 & 1.67 \\
\hline
\end{tabular}

Table 6

Hazard indexes for acute non-carcinogenic risk $(H I)$ caused by combined exposure to different chemicals for various organs and systems without taking background concentrations into account

\begin{tabular}{|l|c|c|c|c|c|c|}
\hline \multirow{2}{*}{\multicolumn{1}{c|}{ Experiment }} & \multicolumn{6}{|c|}{$\begin{array}{c}\text { Additional acute non-carcinogenic risks for specific organs and systems } \\
\text { (hazard index HI) under 4.5-hour exposure }\end{array}$} \\
\cline { 2 - 8 } & $\begin{array}{c}\text { Respiratory } \\
\text { organs }\end{array}$ & $\begin{array}{c}\text { Systemic } \\
\text { impacts }\end{array}$ & $\begin{array}{c}\text { Nervous } \\
\text { system }\end{array}$ & Development & Eyes & Blood \\
\hline Reference group (non-smokers) & - & - & - & - & - & - \\
\hline Cigarette smoking & 26.54 & 24.86 & 0.01 & 16.90 & 4.04 & 16.90 \\
\hline ENDS consumption & 0.72 & 0.74 & 0.001 & 2.96 & 2.00 & 2.96 \\
\hline ETHS consumption & 0.26 & 0.42 & 0.00 & 0.05 & 0.04 & 0.05 \\
\hline
\end{tabular}

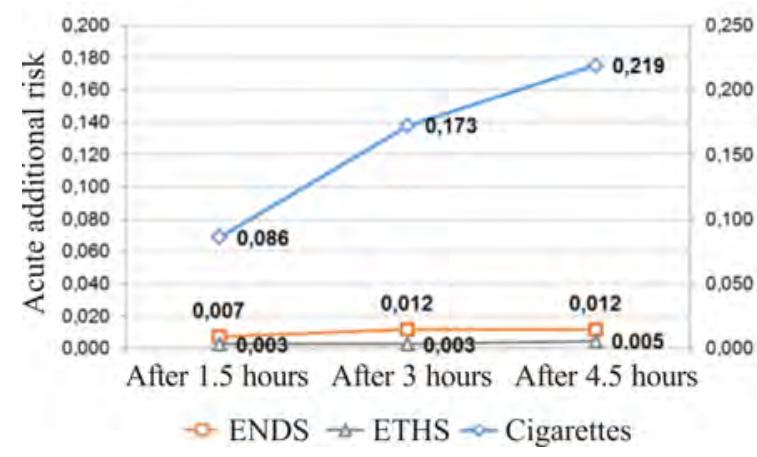

Figure 1. Acute additional risk caused by exposure to benzene for different consumed tobaccoor nicotine-containing products

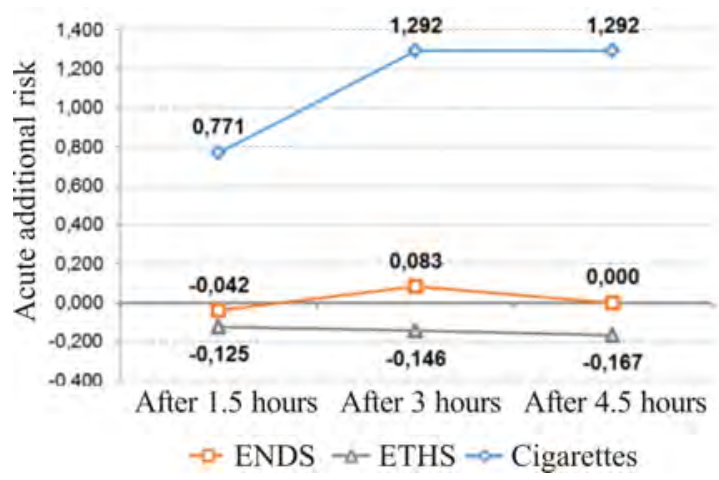

Figure 3. Acute additional risk caused by exposure to formaldehyde for different consumed tobaccoor nicotine-containing products

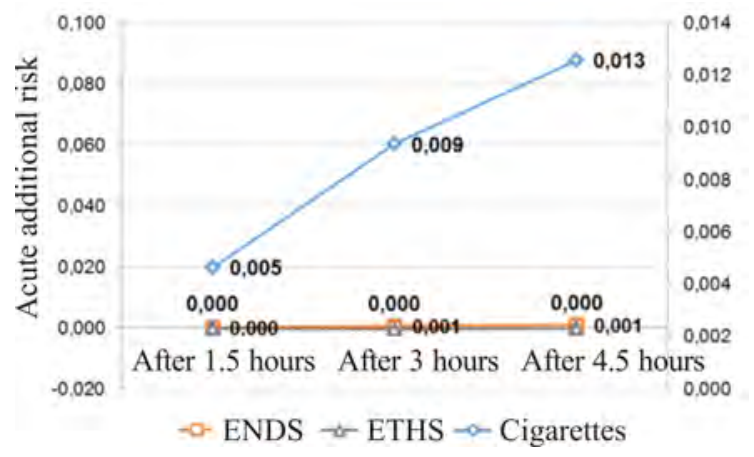

Figure 2. Acute additional risk caused by exposure to toluene for different consumed tobaccoor nicotine-containing products

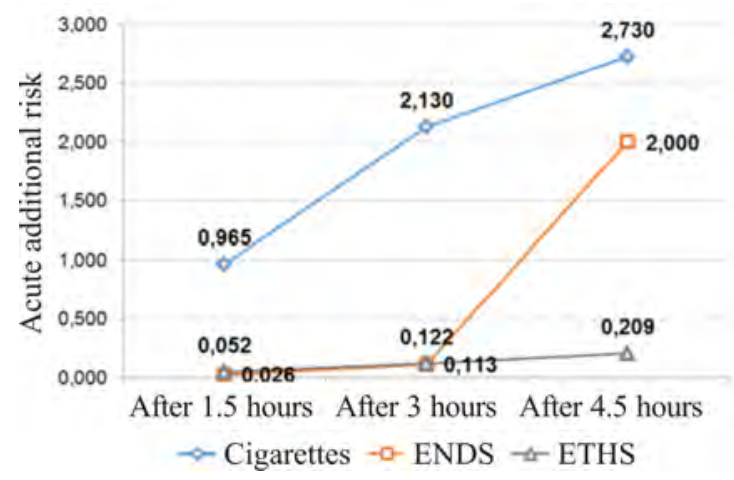

Figure 4. Acute additional risk caused by exposure to acetaldehyde for different consumed tobaccoor nicotine-containing products 


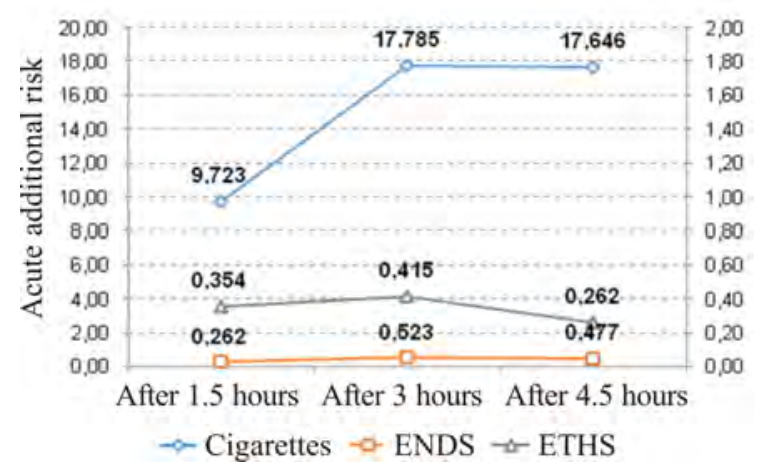

Figure 5. Acute additional risk caused by exposure to particulate matter $\mathrm{PM}_{2,5}$ for different consumed tobacco- or nicotine-containing products

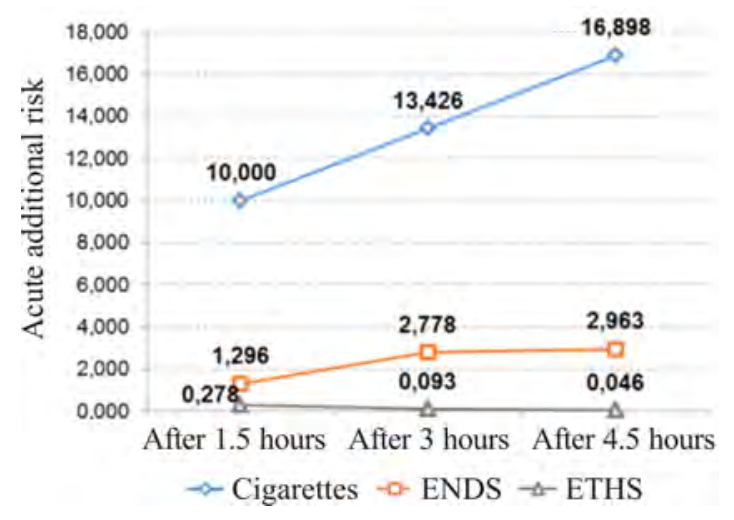

Figure 7. Acute additional risk caused by exposure to carbon oxide for different consumed tobaccoor nicotine-containing products

in adverse chemicals concentrations. But at the same time, this growth in additional risks was the most apparent in case of cigarette smoking as it can be seen in Figures 1-8. This dynamics in additional risk growth was substantially lower in case ENDS or ETHS were used; it was equal to zero or even negative in some cases.

Conclusion. Health risks were assessed basing on the results obtained via laboratory tests performed on the air samples taken inside the closed room under 4.5 -hour exposure that was modeled in the experiment. The assessment revealed that ENDS and ETHS consumption didn't result in any significant changes in air composure and didn't cause any unacceptable acute health risks. When examining combined exposure to contaminants on organs and systems, we established that actual risks were slightly higher than permissible ones for the respiratory organs, eyes, and overall impacts on a body as a whole but they practically didn't differ from

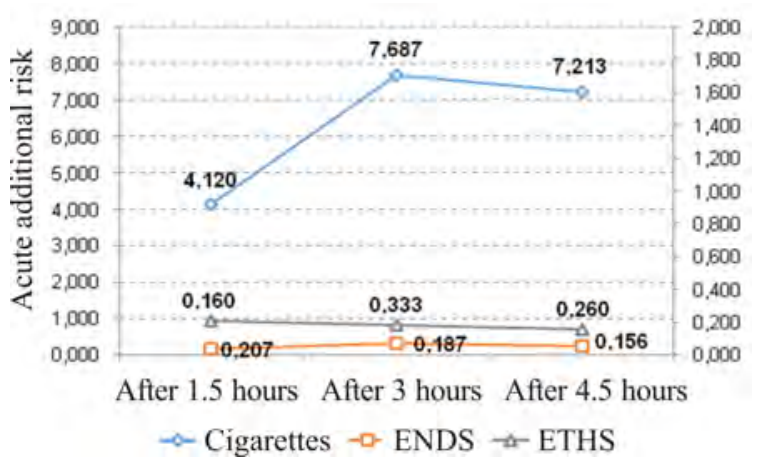

Figure 6. Acute additional risk caused by exposure to particulate matter $\mathrm{PM}_{10}$ for different consumed tobacco- or nicotine-containing products

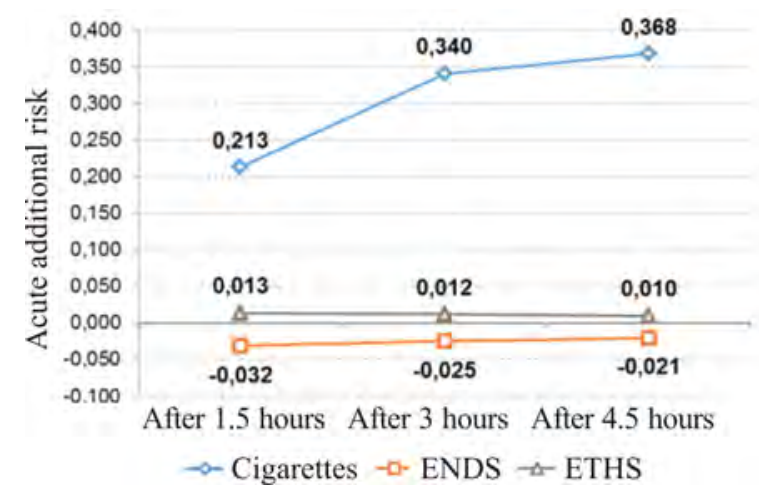

Figure 8. Acute additional risk caused by exposure to nitrogen oxide (II) for different consumed tobaccoor nicotine-containing products

those detected for the reference group. At the same time cigarette smoking caused unacceptable acute risks already 1.5 hours after the experiment started; those risks occurred due to elevated concentrations of acetaldehyde, formaldehyde, particulate matter $\mathrm{PM}_{2,5}$ and $\mathrm{PM}_{10}$, and carbon monoxide. Elevated risk levels caused by ENDS consumption were reached only after 3 hours and occurred due to exposure to only two chemicals, acetaldehyde and carbon oxide.

Our experimental data gave ground for recommending separate places to be organized for ENDS and ETHS consumption and tobacco smoking since ENDS and ETHS consumers may be exposed to additional risks caused by effects produced by tobacco smoke.

Funding. The research was not granted any sponsor support.

Conflict of interests. The authors declare there is no any conflict of interests. 


\section{References}

1. Zubairova L.D., Zubairov D.M. Kurenie kak faktor riska serdechno-sosudistykh zabolevanii [Smoking as a risk factor causing cardiovascular diseases]. Kazanskii meditsinskii zhurnal, 2006, no. 5, pp. 369-373 (in Russian).

2. Krasnova Yu.N. Effects of tobacco smoking on the respiratory system. Sibirskii meditsinskii zhurnal (Irkutsk), 2015, no. 6, pp. 11-15 (in Russian).

3. Tobacco use monitoring and preventive policy. World health organization, 2017. Available at: http://www.who.int/fctc/mediacentre/press-release/wntd-2017/en (27.01.2021).

4. Electronic Nicotine Delivery Systems and Nicotine Delivery Systems (ENDS/EnNDS). Report. World health organization, 2017. Available at: https://www.who.int/tobacco/communications/statements/eletronic-cigarettes-january-2017/en (27.01.2021).

5. Valova A.V., Garipova R.N., Popova O.Yu., Tsapok P.I. Izuchenie vliyaniya osnovnykh komponentov elektronnykh sigaret na organizm cheloveka. Aktual'nye problemy potrebitel'skogo rynka tovarov i uslug [Examining influence exerted by basic components in electronic cigarettes on a human body. Vital issues related to consumer goods and services]. Aktual'nye problemy potrebitel'skogo rynka tovarov i uslug: Materialy IV mezhdunarodnoi zaochnoi nauchno-prakticheskoi konferentsii, posvyashchennoi 30-letiyu Kirovskogo GMU. Kirov, 2017, pp. 31-34 (in Russian).

6. Salagai O.O., Sakharova G.M., Antonov N.S. Electronic nicotine delivery and tobacco heating systems (E-cigarettes): literature review. Narkologiya, 2019, no. 9, pp. 77-100.

7. Bogacheva A.S., Zaritskaya E.V., Yakubova I.Sh., Novikova N.Yu., Laushkin M.A. Kurenie elektronnykh sigaret studentami meditsinskogo VUZa [Electronic cigarettes smoking by students attending a medical HEE]. Profilakticheskaya meditsina - 2019: sbornik nauchnykh trudov Vserossiiskoi nauchno-prakticheskoi konferentsii s mezhdunarodnym uchastiem. Chast' 1. In: A.V. Mel'tser, I.Sh. Yakubova eds. Sankt-Peterburg, Izdatel'stvo SZGMU im. I.I. Mechnikova Publ., 2019, pp. 75-79 (in Russian).

8. Shpak E.I., Galkin A.N., Udal'tsova E.V., Gerasimova T.V. Vliyanie kureniya klassicheskikh, elektronnykh sigaret i kal'yanov na organizm cheloveka, $\mathrm{v}$ tom chisle na polost' rta [Impacts produced by classic cigarettes, e-cigarettes, and hookahs smoking on a human body, including the oral cavity]. Aktual'nye problemy meditsinskoi nauki i obrazovaniya: sbornik statei VI Mezhdunarodnoi nauchnoi konferentsii, 2017, pp. 188-192 (in Russian).

9. Smith M.R., Clark B., Ljudicke F., Schaller J.P., Vanscheeushhijck P., Hoeng J., Peitsch M.C. Evaluation of the Tobacco Heating System 2.2. Part 1: Description of the system and the scientific assessment program. Regul. Toxicol. Pharmacol., 2016, vol. 81, no. 2, pp. S17-S26. DOI: 10.1016/j.yrtph.2016.07.006

10. Baidil'dinova G.K., Mukhanova S.K., Sergazy Sh.D., Mikhalovskii S.V., Gulyaev A.E., Nurgozhin T.S. Estimating a probability of reducing risks associated with smoking conventional cigarettes using the THS2.2 (IQOS) technology. Meditsina (Almaty), 2019, vol. 200, no. 2, pp. 42-50 (in Russian).

11. Mallock N., Bjoss L., Burk R., Danziger M., Shhelsch T., Hahn H., Trieu H.-L., Hahn J. [et al.]. Levels of selected analytes in the emissions of «heat not burn» tobacco products that are relevant to assess human health risks. Archives of Toxicology, 2018, vol. 92, no. 6, pp. 2145-2149. DOI: 10.1007/s00204-018-2215-y

12. Helen G.St., Jacob III P., Nardone N., Benowitz N.L. IQOS: examination of Philip Morris International's claim of reduced exposure. Tobacco control, 2018, no. 27, pp. S30-S36. DOI: 10.1136/tobaccocontrol-2018-054321

13. Zavel'skaya A.Ya., Syrtsova L.E., Levshin V.F. Passive smoking and the risk of cervical cancer. Narkologiya, 2015, vol. 167, no. 11, pp. 52-56 (in Russian).

14. Titova O.N., Kulikov V.D., Sukhovskaya O.A. Passive smoking and respiratory diseases. Meditsinskii al'yans, 2016, no. 3, pp. 73-77 (in Russian).

15. Pokhaznikova M.A., Kuznetsova O.Yu., Lebedev A.K. The prevalence of passive smoking and other risk factors of chronic obstructive pulmonary disease in Saint Petersburg. Rossiiskii semeinyi vrach, 2015, no. 4, pp. 21-28 (in Russian).

16. Mitova M.I., Kampelos P.B., Gujon-Ginlinger K.G., Mader S., Mott'e N., Ruzhe Je.G., Farini M., Triker A.R. Comparison of the impact of the Tobacco Heating System 2.2 and a cigarette on indoor air quality. Regul. Toxicol. Pharmacol., 2016, no. 80, pp. 91-101. DOI: 10.1016/j.yrtph.2016.06.005 
17. Mottier N., Tharin M., Cluse C., Crudo J.-R., Lueso M.G., Goujon-Ginglinger C.G., Jaquier A., Mitova M.I. [et al.]. Validation of selected analytical method using accuracy profiles to assess the impact of Tobacco Heating System on indoor air quality. Talanta, 2016, no. 158, pp. 165-178. DOI: 10.1016/j.talanta.2016.05.022

18. Li H., Luo J., Jiang H., Zhang H., Zhu F., Hu S., Hou H., Hu J., Pang J. Chemical Analysis and Simulated Pyrolysis of Tobacco Heating System 2.2 Compared to Conventional Cigarettes. Nicotine. Tob. Res., 2019, vol. 21, no. 1, pp. 111-118. DOI: 10.1093/ntr/nty005

19. Protano C., Manigrasso M., Avino P., Vitali M. Second-hand smoke generated by combustion and electronic smoking devices used in real scenarios: Ultrafine particle pollution and age-related dose assessment. Environment international, 2017, no. 107, pp. 190-195. DOI: 10.1016/j.envint.2017.07.014

20. Moiseev I.V., Podkopaev D.O., Savin V.M., Leznyi V.V., Prikhod'ko R.P., Simdyanova T.P., Moiseyak M.B., Filatova I.A. [et al.]. Sravnitel'nye issledovaniya komponentnogo sostava sigaret i stikov «Parliament» dlya sistemy nagrevaniya tabaka IQOS [Comparative studies on component structure of «Parliament» cigarettes and tobacco units for IQOS tobacco heating devices]. Mezhdunarodnyi industrial'nyi tabachnyi zhurnal «Tobakko-Revyu», 2017, no. 2 (83), pp. 50-61 (in Russian).

21. Zaritskaya E.V., Yakubova I.Sh., Mikheeva A.Yu., Alikbaeva L.A. Hygienic assessment of chemical composition of pollutants generated in various ways of consumption nicotine-containing product. Gigiena i sanitariya, 2020, vol. 99, no. 6, pp. 638-644 (in Russian).

22. Goniewicz M.L., Knysak J., Gawron M., Kosmider L., Sobczak A., Kurek J., Benowitz N. Levels of selected carcinogens and toxicants in vapour from electronic cigarettes. Tobacco Control, 2014, vol. 23, no. 2, pp. 133-139. DOI: 10.1136/tobaccocontrol-2012-050859

23. Schripp T., Markewitz D., Uhde E., Salthammer T. Does e-cigarette consumption cause passive vaping? Indoor Air, 2013, vol. 23, no. 1, pp. 25-31. DOI: 10.1111/j.1600-0668.2012.00792.x

Zaritskaya E.V., Fedorov V.N., Iakubova I.S. Assessing acute inhalation health risk caused by exposure to products created by nicotine-containing stuff consumption in enclosed spaces. Health Risk Analysis, 2021, no. 2, pp. 62-71. DOI: 10.21668/health.risk/2021.2.06.eng

Received: 29.09 .2020

Accepted: 07.06.2021

Published: 30.09 .2021 